\title{
Cis-Trans Selectivity of Haloalkene Isomers in Nonporous Adaptive Pillararene Crystals
}

Yujuan Zhou, Kecheng Jie, * Run Zhao and Feihe Huang*

State Key Laboratory of Chemical Engineering, Center for Chemistry of

High-Performance \& Novel Materials, Department of Chemistry, Zhejiang University, Hangzhou, Zhejiang 310027, P. R. China; Fax and Tel: +86-571-8795-3189; Email : jiekecheng@zju.edu.cn; fhuang@zju.edu.cn

\section{Supporting Information ( 22 pages)}

1. Materials S2

2. Methods S2

3. Crystallography Data S5

4. Characterization of Desolvated EtP5 Crystals (EtP5 $\alpha$ ) and EtP6 crystals $\begin{array}{ll}(E t P 6 \beta) & \text { S7 }\end{array}$

5. Vapor-Phase Adsorption Measurements $\quad \mathrm{S} 12$

6. Recyclability of EtP6 $\beta$ Crystals $\quad$ S20

$\begin{array}{ll}\text { 7. References } & \text { S22 }\end{array}$ 


\section{Materials}

$p$-Diethoxybenzene was purchased from JK Chemicals and used as received. All other chemicals, including trans-1,4-dichloro-2-butene (trans-DCB) and cis-1,4-dichloro-2-butene (cis-DCB), were purchased from Sigma-Aldrich and used as received. EtP5 and EtP6 were synthesized as described previously. ${ }^{\text {S1 Desolvated }}$ crystalline EtP5 (EtP5 $\alpha$ ) was recrystallized from tetrahydrofuran and dried under vacuum at $60{ }^{\circ} \mathrm{C}$ overnight. Desolvated crystalline EtP6 (EtP6 $\left.\beta\right)$ was recrystallized from acetone and dried under vacuum at $140{ }^{\circ} \mathrm{C}$ overnight. All the mixtures were $v: v=$ $1: 1$.

\section{Methods}

\subsection{Solution NMR}

Solution ${ }^{1} \mathrm{H}$ NMR spectra were recorded at $400.13 \mathrm{MHz}$ using a Bruker Avance 400 NMR spectrometer.

\subsection{Thermogravimetric Analysis}

Thermogravimetric (TGA) analysis was carried out using a Q5000IR analyzer (TA Instruments) with an automated vertical overhead thermobalance. The samples were heated at the rate of $10{ }^{\circ} \mathrm{C} / \mathrm{min}$ using $\mathrm{N}_{2}$ as the protective gas.

\subsection{Powder X-Ray Diffraction}

PXRD data before and after vapor soption were collected on a Rigaku Ultimate-IV $\mathrm{X}$-ray diffractometer operating at $40 \mathrm{kV} / 30 \mathrm{~mA}$ using the $\mathrm{Cu} \mathrm{K \alpha}$ line $(\lambda=1.5418 \AA)$. Data were measured over the range of $5-40^{\circ}$ in $5^{\circ} / \mathrm{min}$ steps over $7 \mathrm{~min}$.

\subsection{Single Crystal Growth}

Single crystals of cis-DCB and trans-DCB loaded EtP5 were grown by slow evaporation: $5 \mathrm{mg}$ of dry EtP5 powders were put in a small vial where $2 \mathrm{~mL}$ of cis-DCB or trans-DCB was added. The resultant transparent solution was allowed to evaporate slowly to give colourless crystals in 5 to 10 days.

Single crystals of cis-DCB and trans-DCB loaded EtP6 were grown by slow evaporation: $5 \mathrm{mg}$ of dry EtP6 powders were put in a small vial where $2 \mathrm{~mL}$ of cis-DCB or trans-DCB was added. The resultant transparent solution was allowed to evaporate slowly to give colourless crystals in 5 to 10 days. 


\subsection{Single Crystal X-ray Diffraction}

Single crystal X-ray data sets were measured on a Rigaku MicroMax-007 HF rotating anode diffractometer (Mo-K $\alpha$ radiation, $\lambda=0.71073 \AA$, Kappa 4-circle goniometer, Rigaku Saturn724+ detector). Unless stated, solvated single crystals, isolated from the crystallization solvent, were immersed in a protective oil, mounted on a MiTeGen loop, and flash cooled under a dry nitrogen gas flow. Empirical absorption corrections, using the multi-scan method, were performed with the program SADABS. ${ }^{\text {S2,S3 }}$ Structures were solved with SHELXD ${ }^{\mathrm{S} 4}$ or SHELXT, ${ }^{\mathrm{S} 5}$ or by direct methods using SHELXS, ${ }^{\mathrm{S} 6}$ refined by full-matrix least squares on $|F|^{2}$ by SHELXL, ${ }^{\mathrm{S} 7}$ and interfaced through the programme OLEX2. ${ }^{\mathrm{S} 8}$ Unless stated, all non-H-atoms were refined anisotropically, and all $\mathrm{H}$-atoms were fixed in geometrically estimated positions and refined using the riding model. Supplementary CIFs, which include structure factors, are available free of charge from the Cambridge Crystallographic Data Centre (CCDC) via www.ccdc.cam.ac.uk/data_request/cif.

\subsection{Gas Chromatography}

Gas chromatography (GC) analysis: GC measurements were carried out using an Agilent 7890B instrument configured with an FID detector and a DB-624 column (30 $\mathrm{m} \times 0.53 \mathrm{~mm} \times 3.0 \mu \mathrm{m})$. Samples were analyzed using headspace injections and were performed by incubating the sample at $150{ }^{\circ} \mathrm{C}$ for 10 minutes followed by sampling 1 $\mathrm{mL}$ of the headspace. The following GC method was used: the oven was programmed from $50{ }^{\circ} \mathrm{C}$, ramped at $10{ }^{\circ} \mathrm{C} \mathrm{min}{ }^{-1}$ increments to $150{ }^{\circ} \mathrm{C}$ with 15 min hold; the total run time was $25 \mathrm{~min}$; injection temperature $250{ }^{\circ} \mathrm{C}$; detector temperature $280{ }^{\circ} \mathrm{C}$ with hydrogen, air, and make-up flow-rates of 35,350 , and $35 \mathrm{~mL} \mathrm{~min}{ }^{-1}$, respectively; helium (carrier gas) flow-rate $3.0 \mathrm{~mL} \mathrm{m^{-1 }}$. The samples were injected in the split mode (30:1).

\subsection{Gas Sorption Measurement}

Low-pressure gas adsorption measurements were performed on a Micrometritics Accelerated Surface Area and Porosimetry System (ASAP) 2020 surface area analyzer. Samples were degassed under dynamic vacuum for $12 \mathrm{~h}$ at $60{ }^{\circ} \mathrm{C}$ prior to each measurement. $\mathrm{N}_{2}$ isotherms were measured using a liquid nitrogen bath $(77 \mathrm{~K})$.

\subsection{Vapor Sorption Measurement}

Cis-DCB and trans-DCB sorption isotherms were measured via Micromeritics 3Flex. Samples were degassed under dynamic vacuum for $12 \mathrm{~h}$ at $120{ }^{\circ} \mathrm{C}$ prior to each 
measurement. These isotherms were collected at $25{ }^{\circ} \mathrm{C}$ by monitoring the volume change. 


\section{Crystallography Data}

Table S1. Experimental single crystal X-ray data for EtP5 and EtP6 structures.

\begin{tabular}{|c|c|c|c|c|}
\hline & cis-DCB@EtP5 & trans-DCB@EtP5 & 2(cis-DCB)@EtP6 & trans-DCB@EtP6 \\
\hline Crystallisation Solvent & cis-DCB & trans-DCB & cis-DCB & trans-DCB \\
\hline Collection Temperature & $170 \mathrm{~K}$ & $170 \mathrm{~K}$ & $110 \mathrm{~K}$ & $111 \mathrm{~K}$ \\
\hline Formula & $\mathrm{C}_{59} \mathrm{H}_{76} \mathrm{Cl}_{2} \mathrm{O}_{10}$ & $\mathrm{C}_{59} \mathrm{H}_{76} \mathrm{Cl}_{2} \mathrm{O}_{10}$ & $\mathrm{C}_{74} \mathrm{H}_{96} \mathrm{Cl}_{4} \mathrm{O}_{12}$ & $\mathrm{C}_{70} \mathrm{H}_{90} \mathrm{Cl}_{2} \mathrm{O}_{12}$ \\
\hline$M r$ & 1016.09 & 1016.09 & 1319.36 & 1194.36 \\
\hline Crystal Size [mm] & $0.2 \times 0.15 \times 0.12$ & $0.08 \times 0.06 \times 0.05$ & $0.12 \times 0.1 \times 0.08$ & $0.1 \times 0.03 \times 0.02$ \\
\hline Crystal System & Orthorhombic & Orthorhombic & Monoclinic & Monoclinic \\
\hline Space Group & Pbcn & Pbcn & $C 2 / c$ & $P 2{ }_{1} / n$ \\
\hline$a[\AA]$ & $42.9147(15)$ & $43.026(2)$ & $26.441(4)$ & $11.3650(3)$ \\
\hline$b[\AA]$ & $15.7637(5)$ & $15.8064(8)$ & $12.5644(18)$ & $13.1318(3)$ \\
\hline$c[\AA]$ & $16.3301(5)$ & $26.2928(8)$ & $25.776(4)$ & $21.8808(5)$ \\
\hline \multicolumn{5}{|l|}{$\alpha\left[^{\circ}\right]$} \\
\hline$\beta\left[^{\circ}\right]$ & & & $120.301(8)$ & $91.2740(10)$ \\
\hline \multicolumn{5}{|l|}{$\gamma\left[{ }^{\circ}\right]$} \\
\hline$V\left[\AA^{3}\right]$ & $11047.2(6)$ & $11080.6(0)$ & $7393(2)$ & $32644.75(14)$ \\
\hline$Z$ & 8 & 8 & 4 & 4 \\
\hline $\mathrm{D}_{\text {calcd }}\left[\mathrm{g} \mathrm{cm}^{-3}\right]$ & 1.222 & 1.218 & 1.202 & 1.215 \\
\hline$\mu\left[\mathrm{mm}^{-1}\right]$ & 0.983 & 0.98 & 1.036 & \\
\hline $\mathrm{F}(000)$ & 4352 & 4352 & 2868 & 1280 \\
\hline $2 \theta$ range $\left[{ }^{\circ}\right]$ & $7.01-109.99$ & $7.01-109.77$ & $6.66-109.95$ & $6.84-109.84$ \\
\hline Reflections collected & 90616 & 66299 & 7058 & 32219 \\
\hline $\begin{array}{l}\text { Independent reflections, } \\
R_{\text {int }}\end{array}$ & $10505,0.0445$ & $10510,0.0374$ & $7058,0.0465$ & $6170,0.0587$ \\
\hline Obs. Data $[I>2 \sigma(I)]$ & 6390 & 7265 & 6298 & 5129 \\
\hline Data /restraints / & 10505 / $31 /$ & $10510 / 2 /$ & $7058 / 112 /$ & $6170 / 0 /$ \\
\hline parameters & 686 & 650 & 467 & 385 \\
\hline $\begin{array}{l}\text { Final } R_{1} \text { values }(I> \\
2 \sigma(I))\end{array}$ & 0.0715 & 0.0680 & 0.1292 & 0.0627 \\
\hline Final $R_{1}$ values (all data) & 0.1229 & 0.1004 & 0.1361 & 0.0731 \\
\hline $\begin{array}{l}\text { Final } w R\left(F_{2}\right) \text { values (all } \\
\text { data) }\end{array}$ & 0.1850 & 0.1902 & 0.2869 & 0.1733 \\
\hline Goodness-of-fit on $F^{2}$ & 1.039 & 1.054 & 1.089 & 1.032 \\
\hline $\begin{array}{l}\text { Largest difference peak } \\
\text { and hole }\left[\mathrm{e} \cdot \mathrm{A}^{-3}\right]\end{array}$ & $0.573 /-0.468$ & $0.892 /-0.536$ & $2.37 /-1.263$ & $0.579 /-0.76$ \\
\hline $\mathrm{CCDC}$ & 1916689 & 1916690 & 1916691 & 1916692 \\
\hline
\end{tabular}



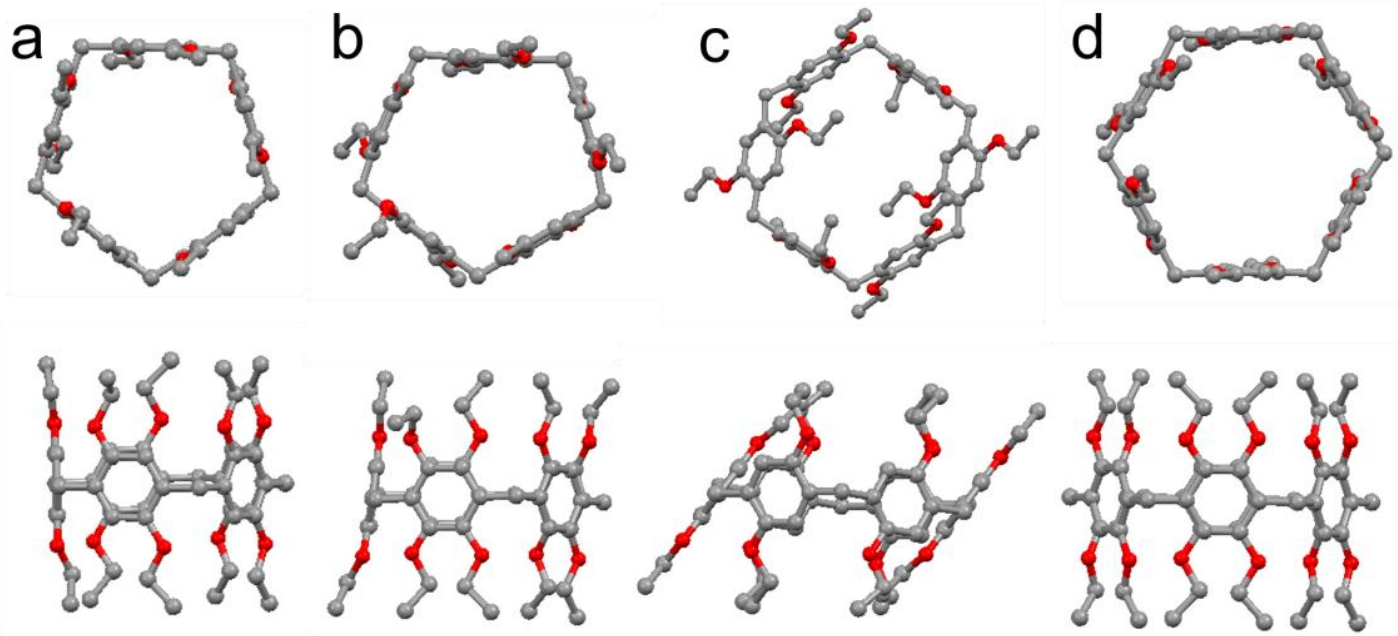

Figure S1. Ball-stick plots from single crystal structures: (a) cis-DCB@EtP5, (b) trans-DCB@EtP5, (c)trans-DCB@EtP6 and (d) 2(cis-DCB)@EtP6; shown in the cavity (top) and in-plane of the aromatic core (bottom). H-atoms and solvent molecules are omitted for clarity. Not shown on a common scale. 
4. Characterization of Desolvated EtP5 Crystals (EtP5 $\alpha$ ) and EtP6 crystals $(\operatorname{EtP6} \beta)$

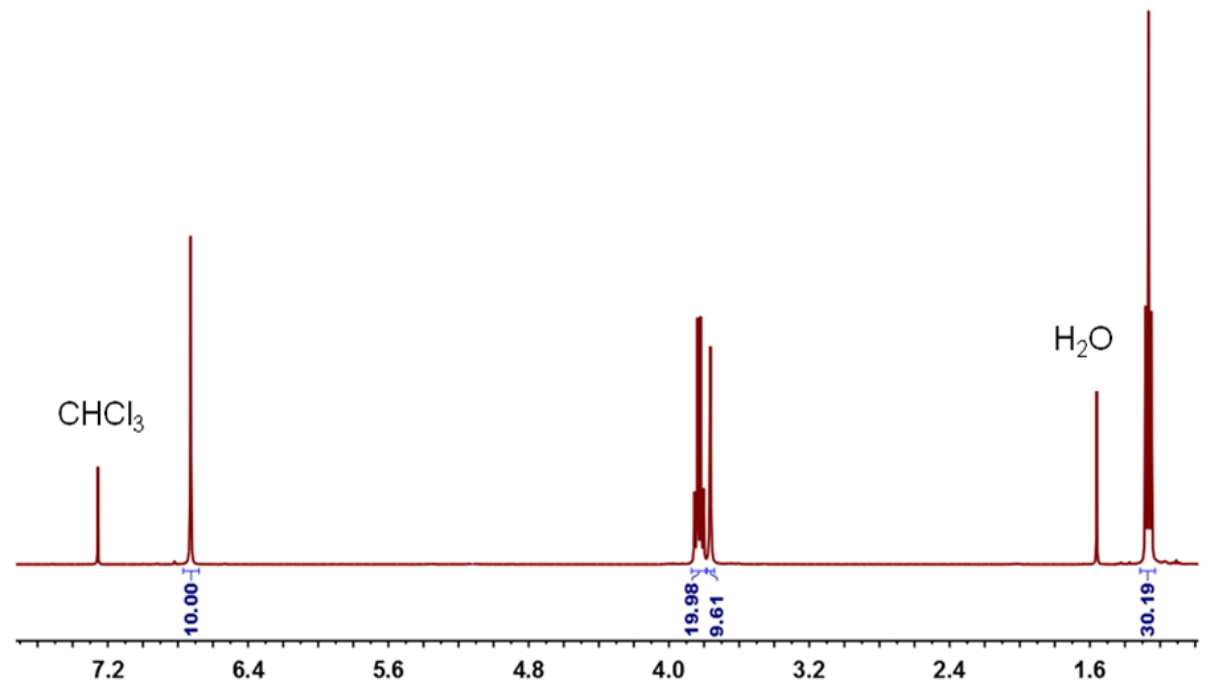

Figure S2. ${ }^{1} \mathrm{H}$ NMR spectrum $\left(400 \mathrm{MHz}, \mathrm{CDCl}_{3}, 293 \mathrm{~K}\right.$ ) of EtP5 $\alpha$. The $\mathrm{H}_{2} \mathrm{O}$ peak is from $\mathrm{CDCl}_{3}$.

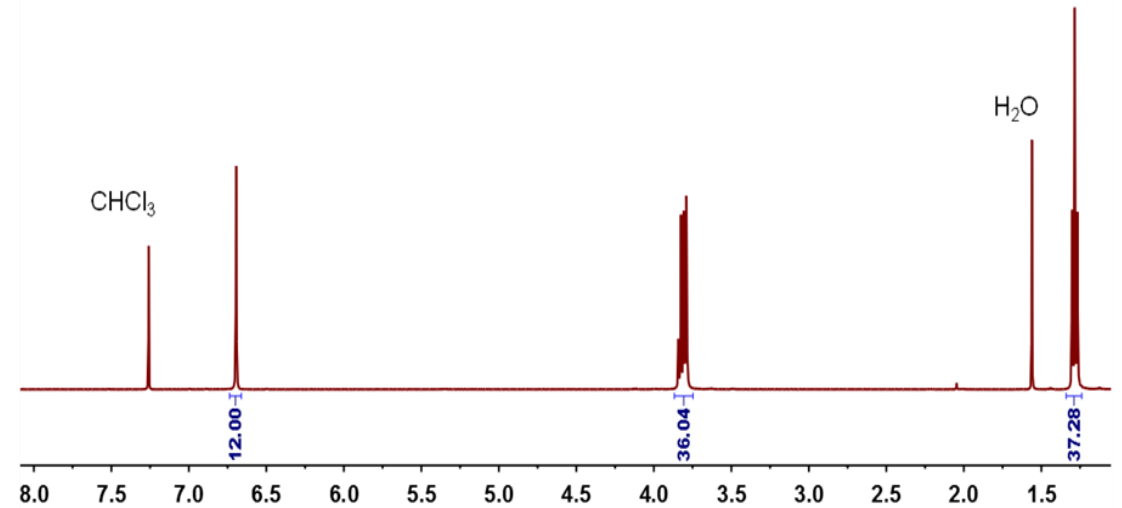

Figure S3. ${ }^{1} \mathrm{H}$ NMR spectrum $\left(400 \mathrm{MHz}, \mathrm{CDCl}_{3}, 293 \mathrm{~K}\right)$ of EtP6 $\beta$. The $\mathrm{H}_{2} \mathrm{O}$ peak is from $\mathrm{CDCl}_{3}$. 


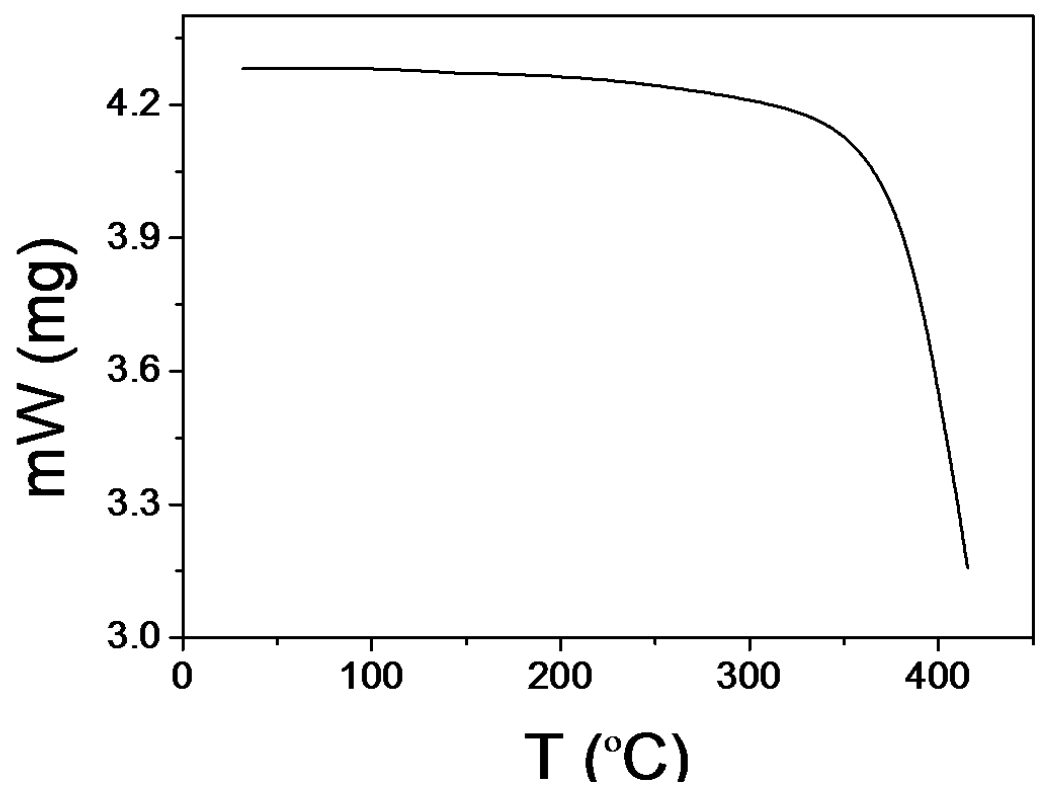

Figure S4. Thermogravimetric analysis of desolvated EtP5.

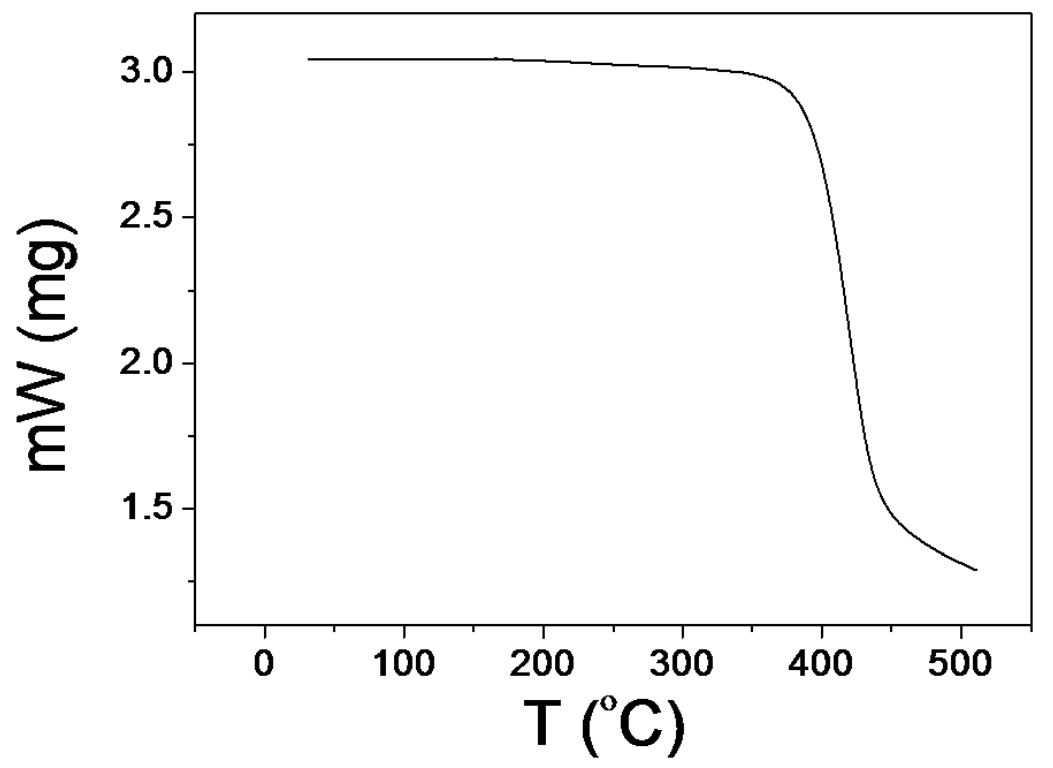

Figure S5. Thermogravimetric analysis of desolvated EtP6. 


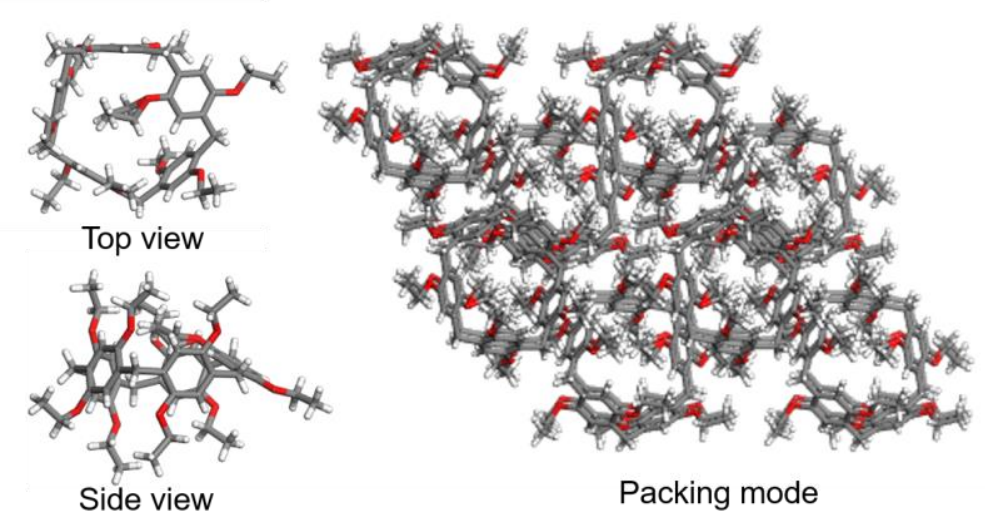

Figure S6. Single crystal structure of guest-free EtP5, which is defined as EtP5 $\alpha .{ }^{\mathrm{S} 9}$

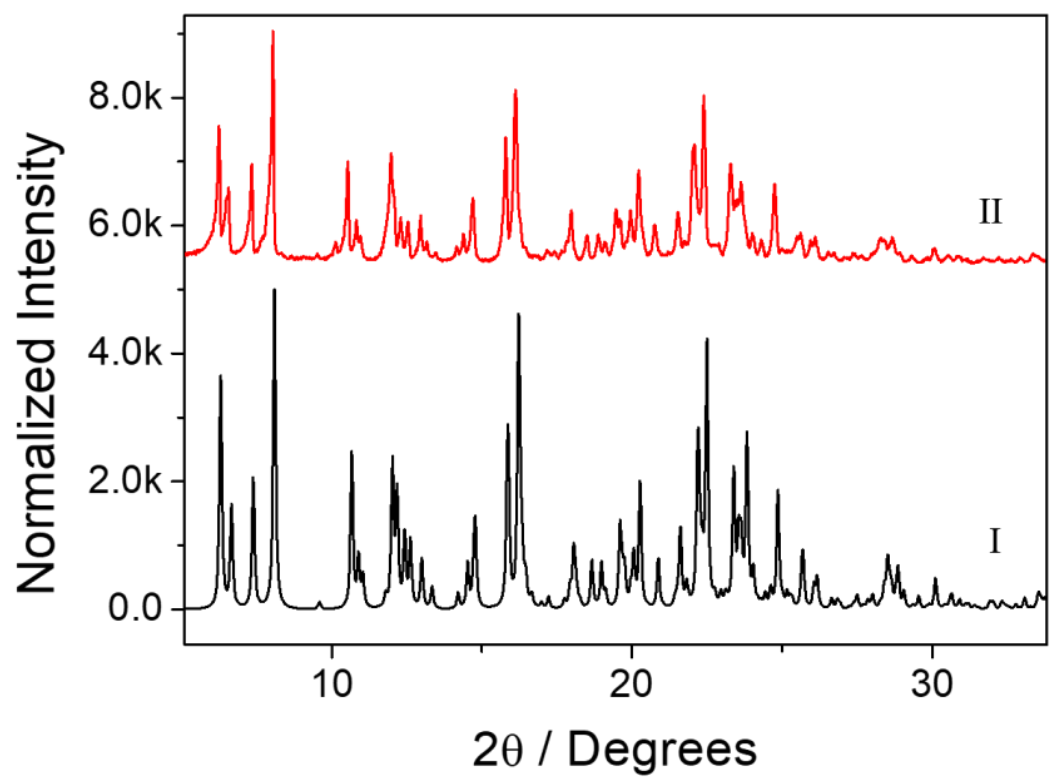

Figure S7. Powder X-ray diffraction patterns: (I) simulated from single crystal structure of guest-free EtP5; (II) activated EtP5 crystals (EtP5 $\alpha$ ). 


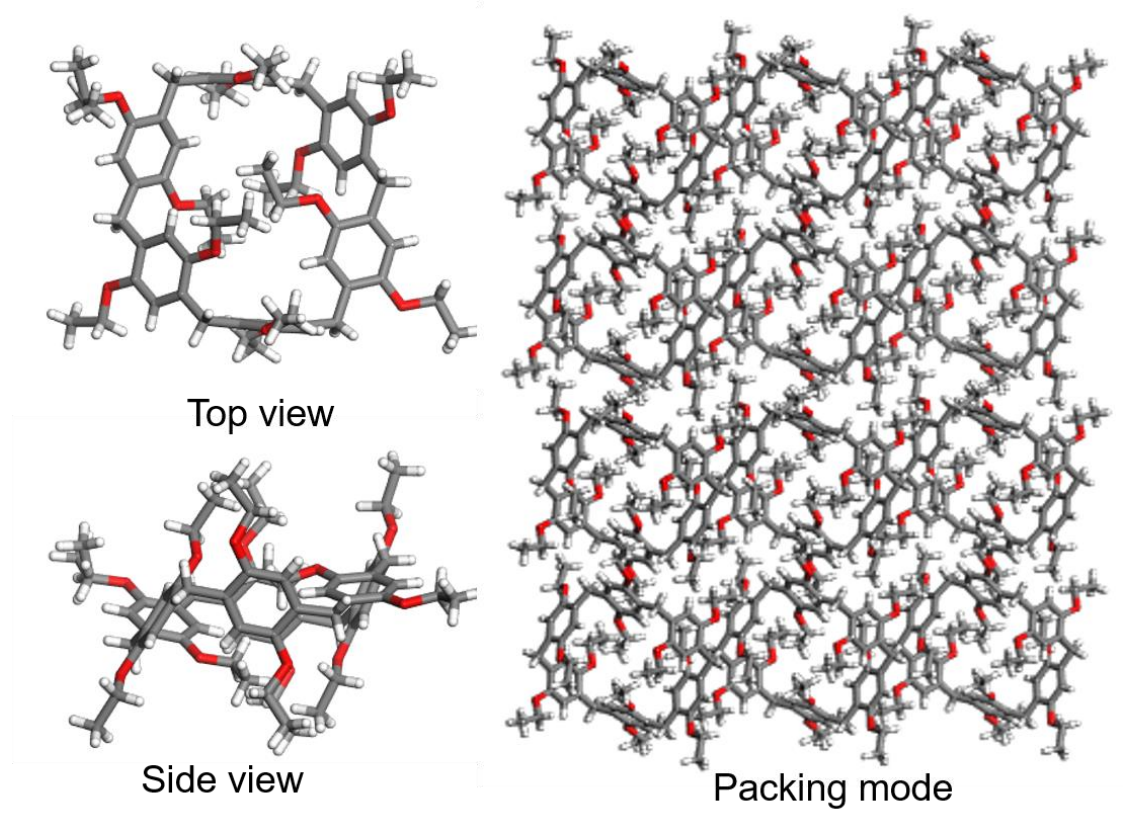

Figure S8. Single crystal structure of thermally stable guest-free EtP6, which is defined as EtP6 $\beta{ }^{\text {S9 }}$

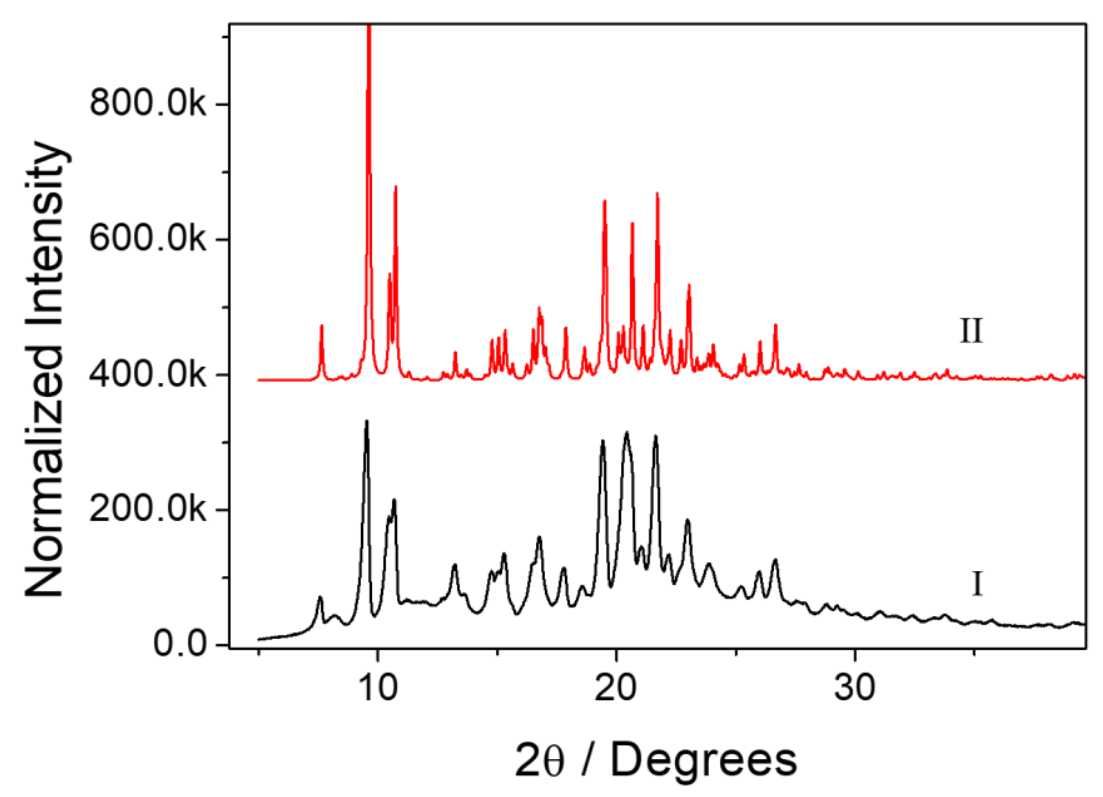

Figure S9. Powder X-ray diffraction patterns: (I) activated EtP6 crystals (EtP6 $\beta$ ); (II) simulated from single crystal structure of guest-free EtP6. 


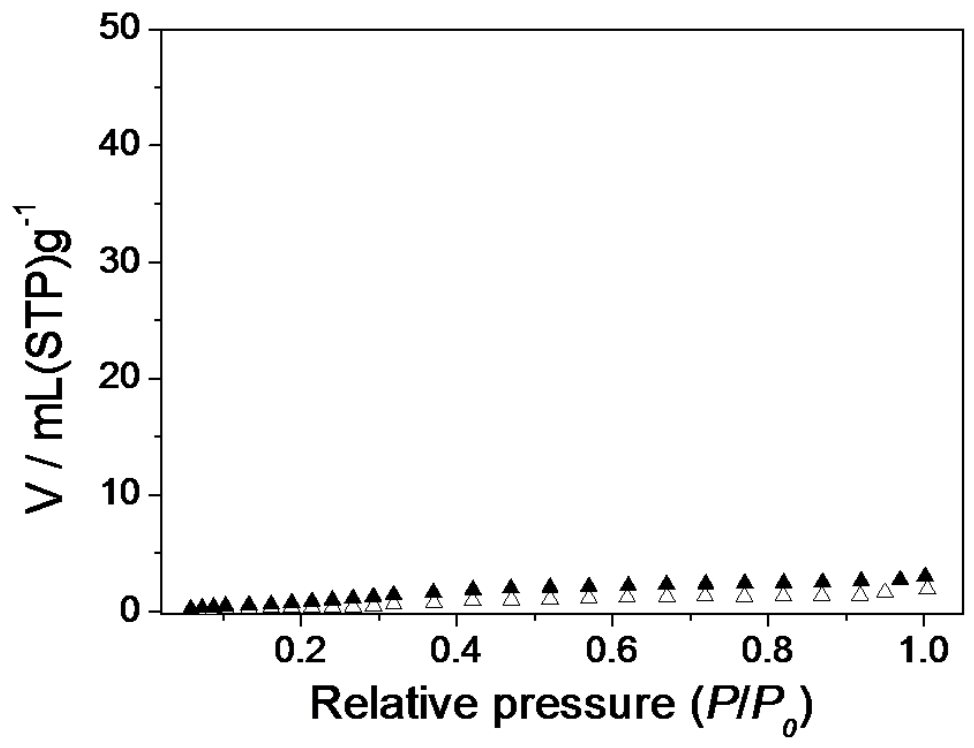

Figure S10. $\mathrm{N}_{2}$ adsorption isotherm of EtP5 $\alpha$. Adsorption, closed symbols; desorption, open symbols.

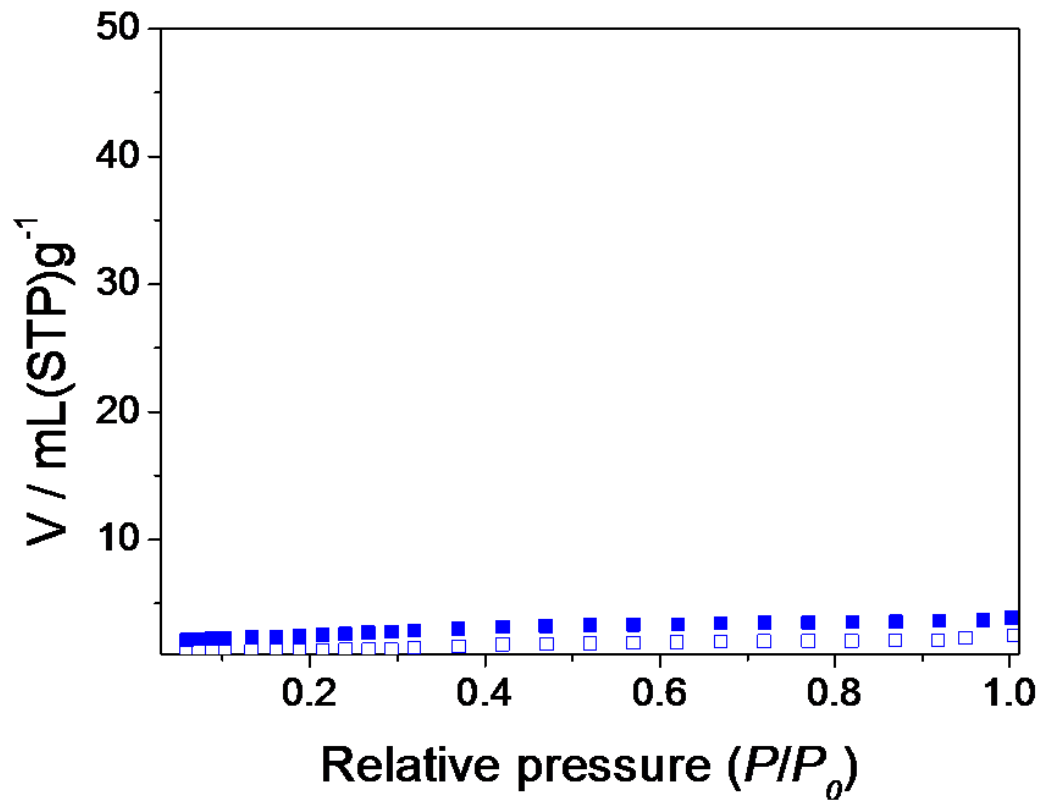

Figure S11. $\mathrm{N}_{2}$ adsorption isotherm of EtP6 $\beta$. Adsorption, closed symbols; desorption, open symbols. 


\section{Vapor-Phase Adsorption Measurements}

\subsection{Single-Component cis-trans DCB Isomer Adsorption Experiments in EtP5 $\alpha$}

${ }^{1} \mathrm{H}$ NMR experiments were performed by dissolving the EtP5 $\alpha$ crystals after vapor sorption in $\mathrm{CDCl}_{3}$. TGA profiles were recorded using EtP5 $\alpha$ crystals after vapor sorption.

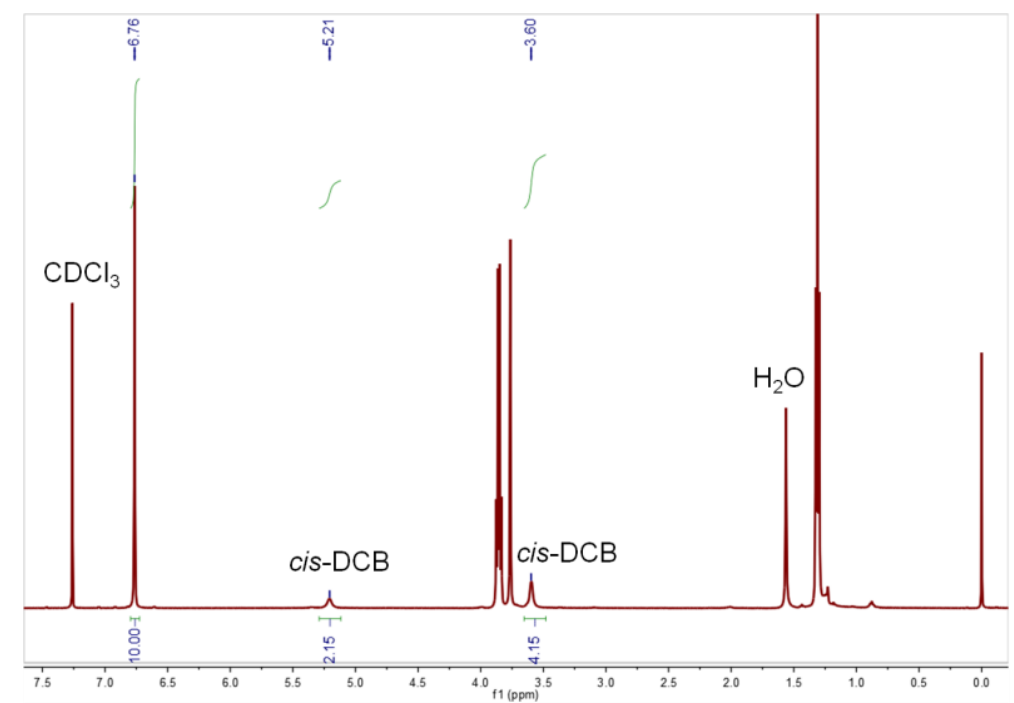

Figure S12. ${ }^{1} \mathrm{H}$ NMR spectrum $\left(400 \mathrm{MHz}, \mathrm{CDCl}_{3}, 293 \mathrm{~K}\right)$ of EtP5 $\alpha$ after adsorption of $c i s$-DCB vapor.

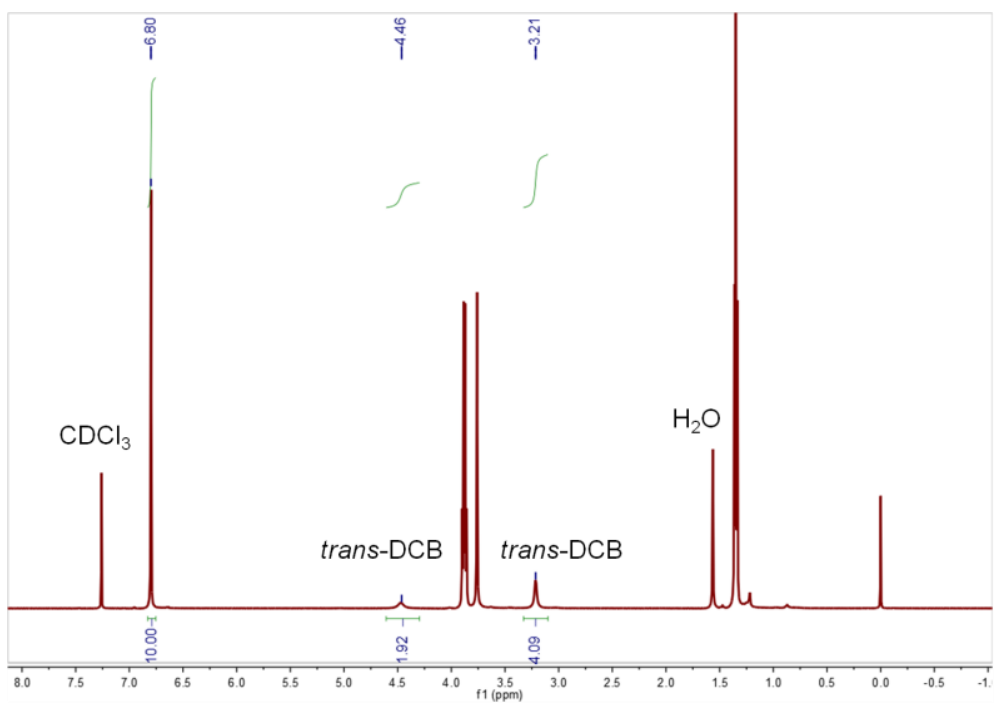

Figure S13. ${ }^{1} \mathrm{H}$ NMR spectrum $\left(400 \mathrm{MHz}, \mathrm{CDCl}_{3}, 293 \mathrm{~K}\right)$ of EtP5 $\alpha$ after adsorption of trans-DCB vapor. 


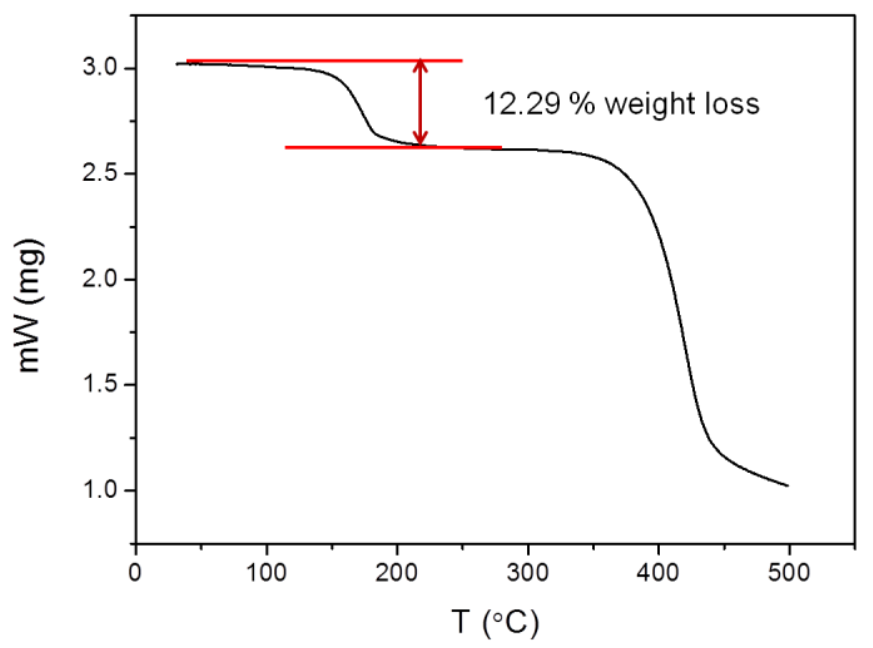

Figure S14. Thermogravimetric analysis of EtP5 $\alpha$ after adsorption of cis-DCB. The weight loss below $200{ }^{\circ} \mathrm{C}$ can be calculated as 1 cis-DCB molecule per EtP5 molecule.

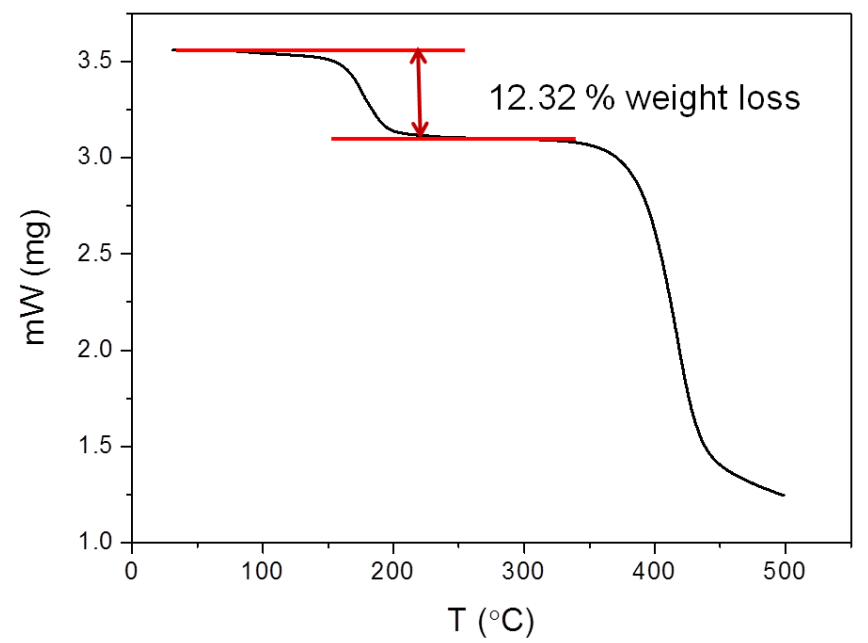

Figure S15. Thermogravimetric analysis of EtP5 $\alpha$ after adsorption of trans-DCB. The weight loss below $200{ }^{\circ} \mathrm{C}$ can be calculated as 1 trans-DCB molecule per EtP5 molecule. 


\subsection{Single-Component cis-trans DCB Isomer Adsorption Experiments in EtP6 $\beta$}

${ }^{1} \mathrm{H}$ NMR experiments were performed by dissolving EtP6 $\beta$ crystals after vapor sorption. TGA profiles were recorded using EtP6 $\beta$ crystals after vapor sorption.

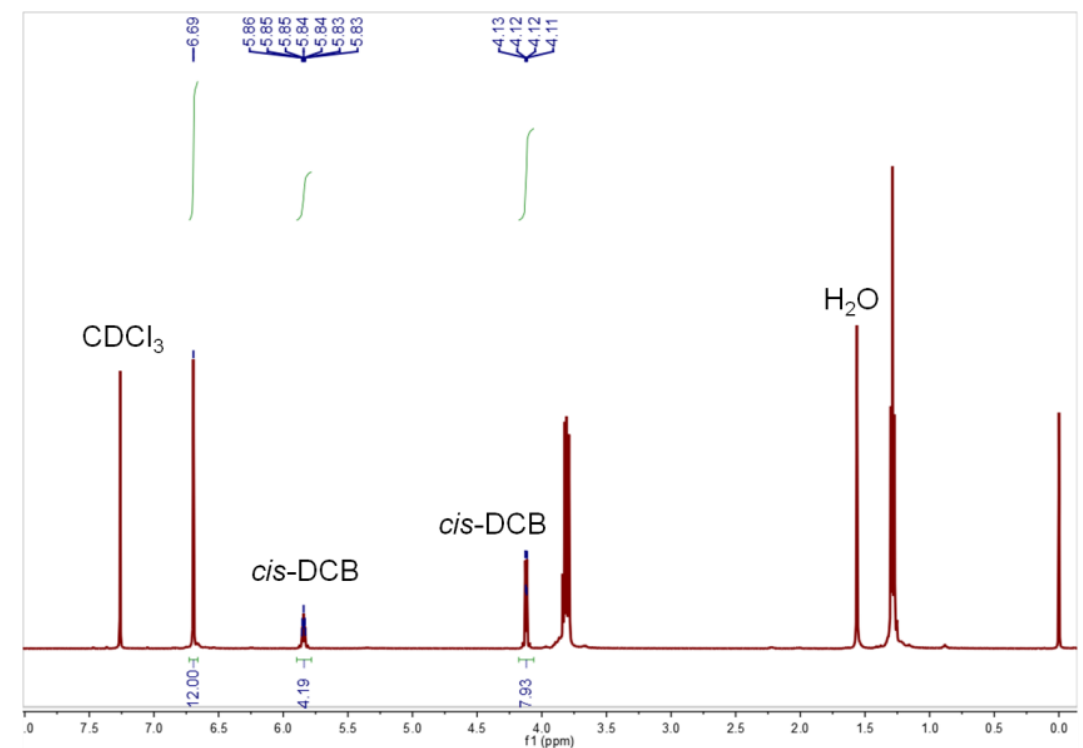

Figure S16. ${ }^{1} \mathrm{H}$ NMR spectrum $\left(400 \mathrm{MHz}, \mathrm{CDCl}_{3}, 293 \mathrm{~K}\right)$ of EtP6 $\beta$ after adsorption of $c i s$-DCB vapor.

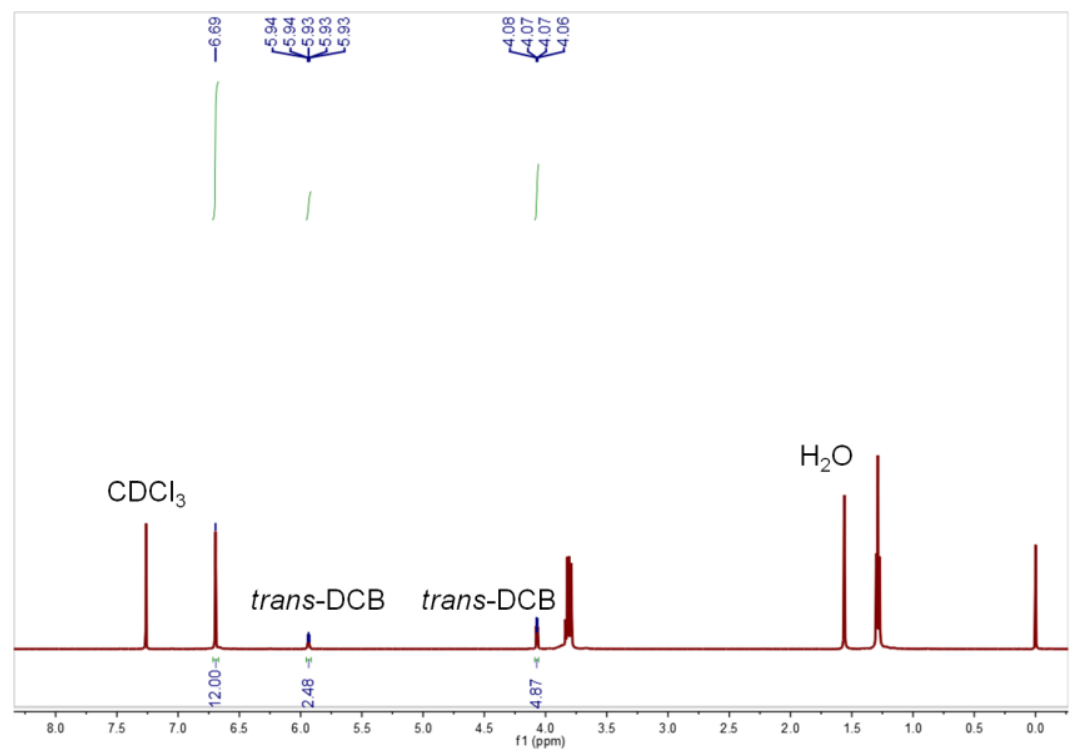

Figure S17. ${ }^{1} \mathrm{H}$ NMR spectrum $\left(400 \mathrm{MHz}, \mathrm{CDCl}_{3}, 293 \mathrm{~K}\right)$ of EtP6 $\beta$ after adsorption of trans-DCB vapor. 


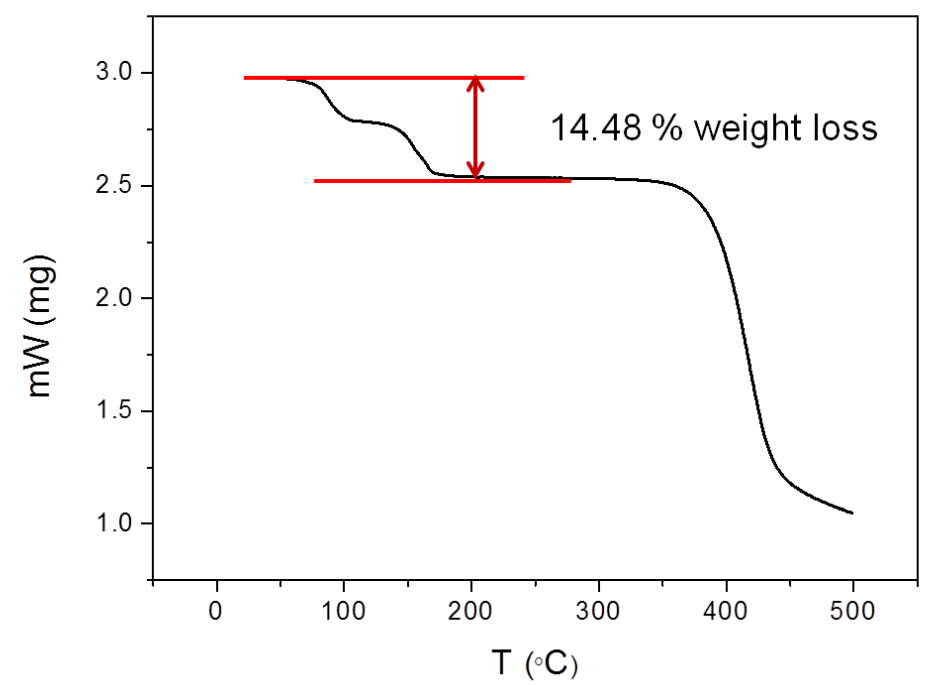

Figure S18. Thermogravimetric analysis of EtP6 $\beta$ after adsorption of cis-DCB. The weight loss below $170{ }^{\circ} \mathrm{C}$ can be calculated as 2.05 cis-DCB molecules per EtP6 molecule.

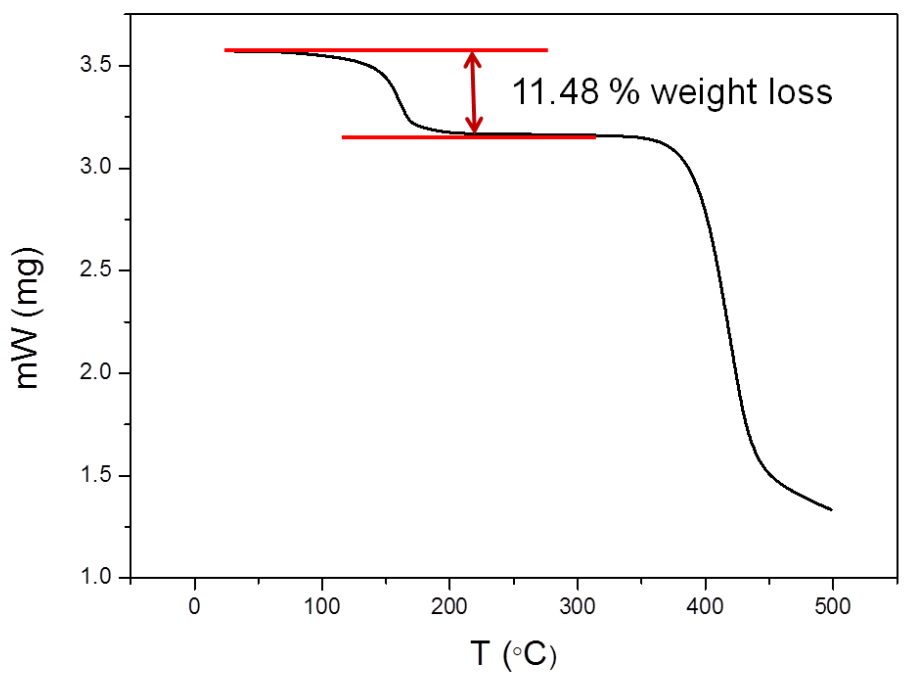

Figure S19. Thermogravimetric analysis of EtP6 $\beta$ after adsorption of trans-DCB. The weight loss below $200{ }^{\circ} \mathrm{C}$ can be calculated as 1 trans-DCB molecule per EtP6 molecule. 


\subsection{Structural Analyses after Single-Component Vapor Sorption}

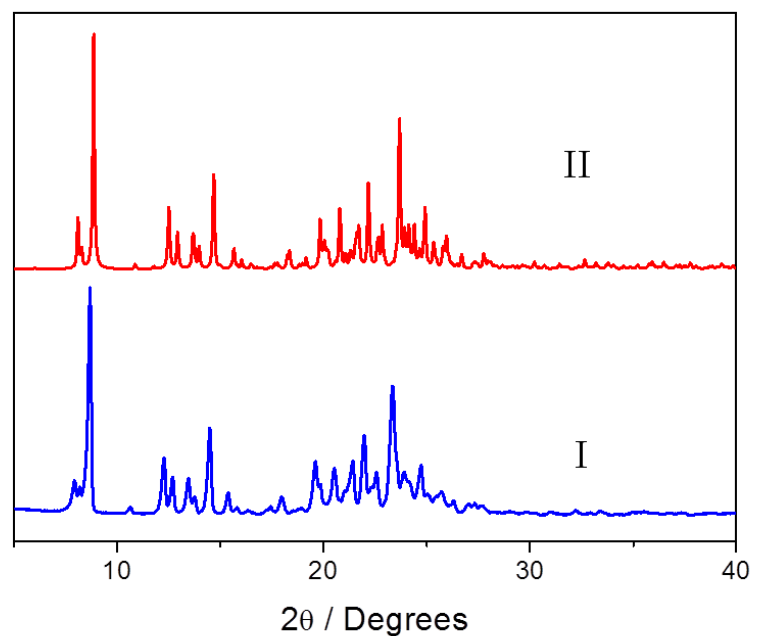

Figure S20. Powder X-ray diffraction patterns of EtP5: (I) EtP5 $\alpha$ after adsorption of cis-DCB; (II) simulated from single crystal structure of cis-DCB@EtP5.

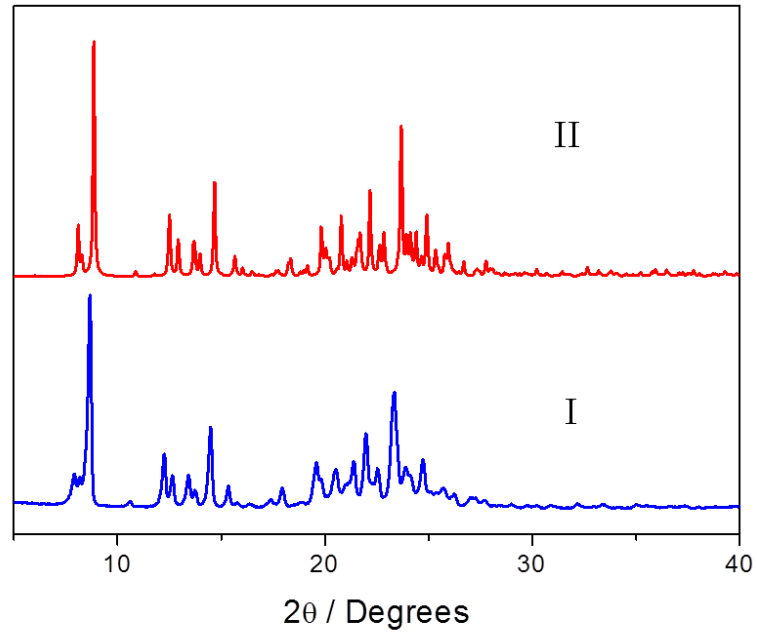

Figure S21. Powder X-ray diffraction patterns of EtP5: (I) EtP5 $\alpha$ after adsorption of trans-DCB; (II) simulated from single crystal structure of trans-DCB @EtP5. 


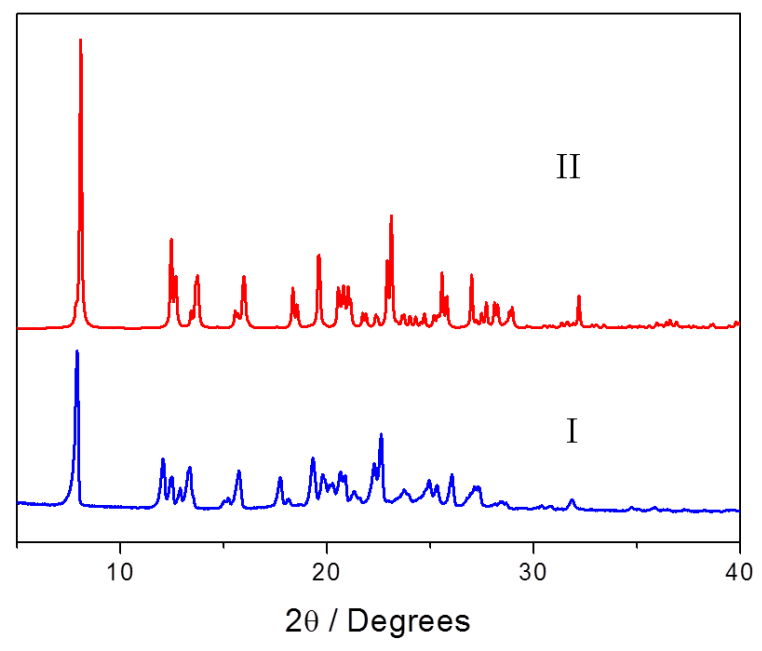

Figure S22. Powder X-ray diffraction patterns of EtP6: (I) EtP6 $\beta$ after adsorption of cis-DCB; (II) simulated from single crystal structure of 2(cis-DCB)@EtP6.

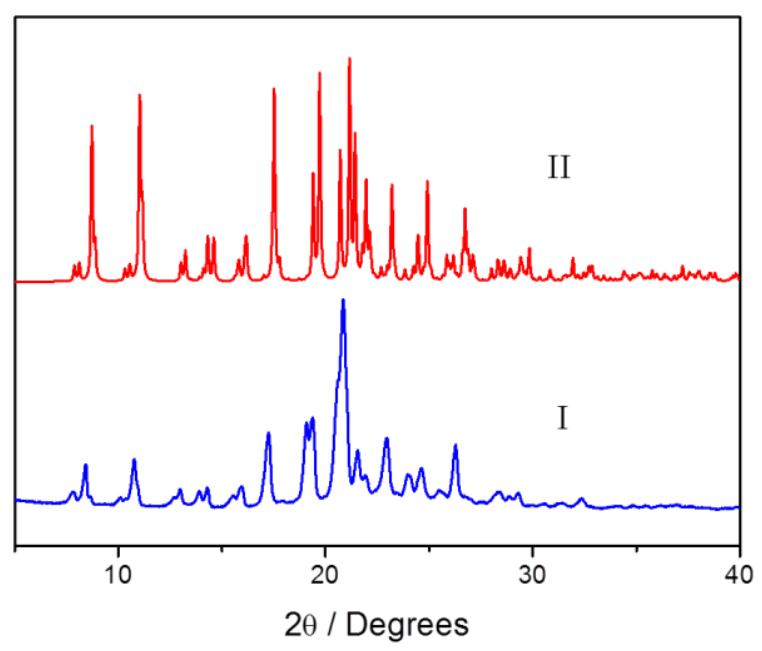

Figure S23. Powder X-ray diffraction patterns of EtP6: (I) EtP6 $\beta$ after adsorption of trans-DCB; (II) simulated from single crystal structure of trans-DCB @EtP6. 


\subsection{Uptake from a cis-trans DCB Isomer Mixture in EtP5 $\alpha$ and EtP6 $\beta$}

For each mixture vapor-phase experiment, an open $5 \mathrm{~mL}$ vial containing $0.020 \mathrm{~g}$ of guest-free EtP5 $\alpha$ or EtP6 $\beta$ adsorbent was placed in a sealed $20 \mathrm{~mL}$ vial containing 2 $\mathrm{mL}$ of a cis-trans DCB mixture $(v: v=1: 1)$. Uptake in the EtP5 $\alpha$ or EtP6 $\beta$ crystals was measured hour by hour by completely dissolving the crystals and measuring the ratio of cis-DCB or trans-DCB to EtP5 or EtP6 by ${ }^{1} \mathrm{H}$ NMR. The relative uptake of cis-DCB and trans-DCB in EtP5 $\alpha$ or EtP6 $\beta$ was also measured by heating the crystals to release the adsorbed vapor using gas chromatography (headspace method).

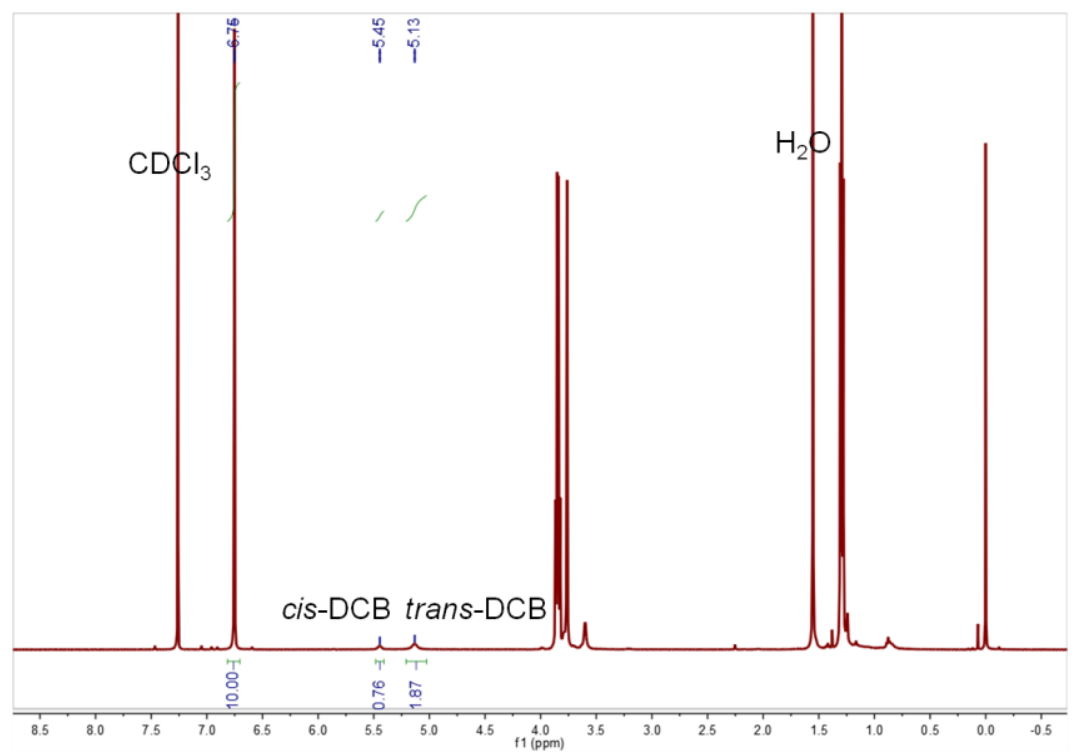

Figure S24. ${ }^{1} \mathrm{H}$ NMR spectrum (400 $\mathrm{MHz}, \mathrm{CDCl}_{3}, 293 \mathrm{~K}$ ) of EtP5 $\alpha$ after sorption of cis-trans DCB mixture vapor.

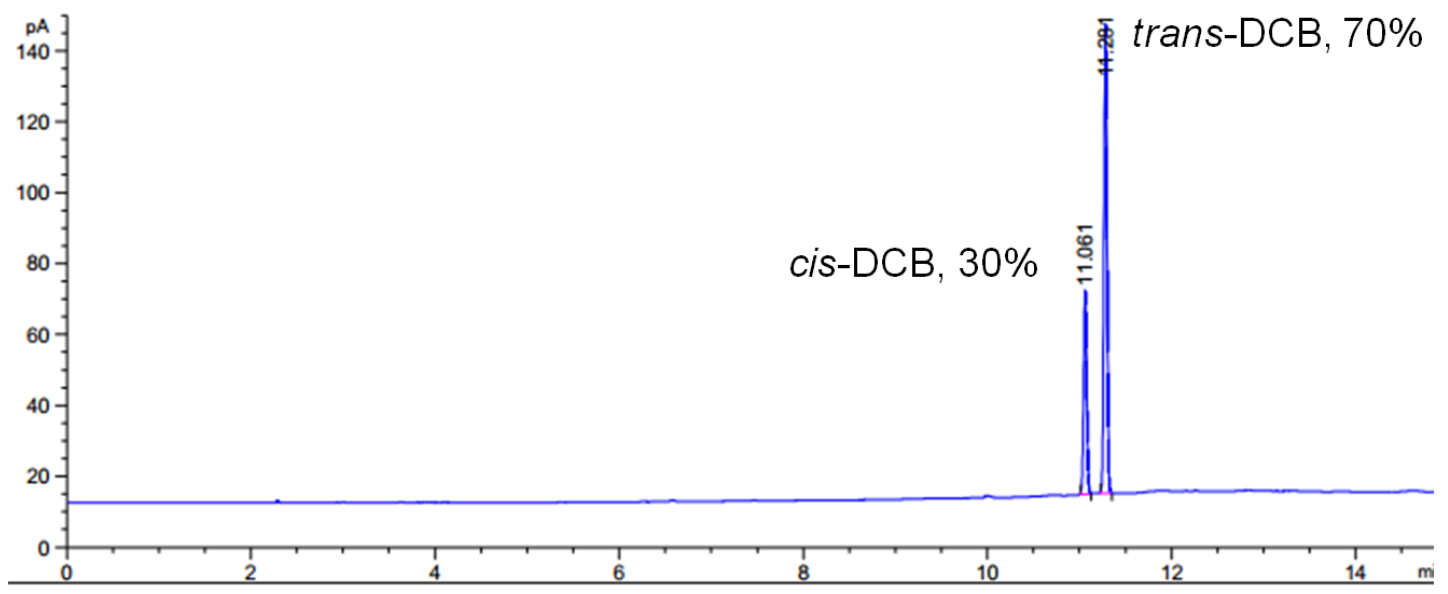

Figure S25. Relative uptake of cis-DCB and trans-DCB adsorbed in EtP5 $\alpha$ after 24 hour using gas chromatography. 


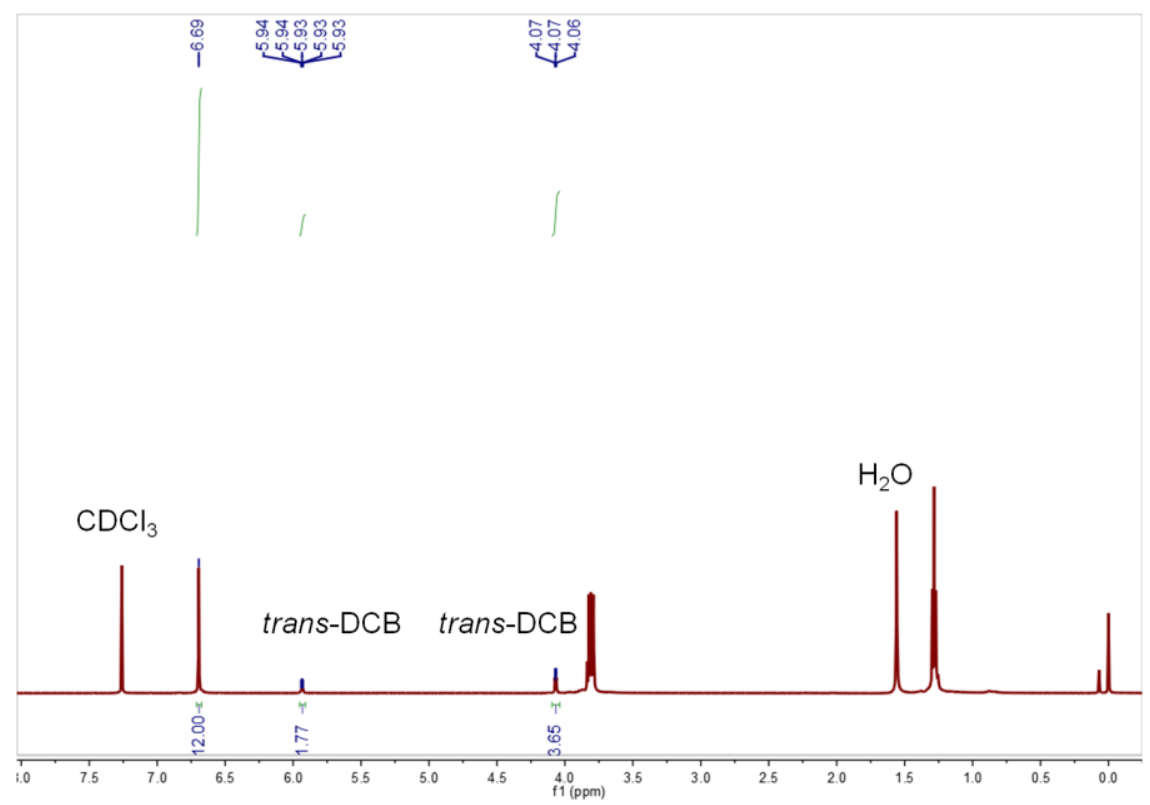

Figure S26. ${ }^{1} \mathrm{H}$ NMR spectrum $\left(400 \mathrm{MHz}, \mathrm{CDCl}_{3}, 293 \mathrm{~K}\right)$ of EtP6 $\beta$ after sorption of cis-trans DCB mixture vapor.

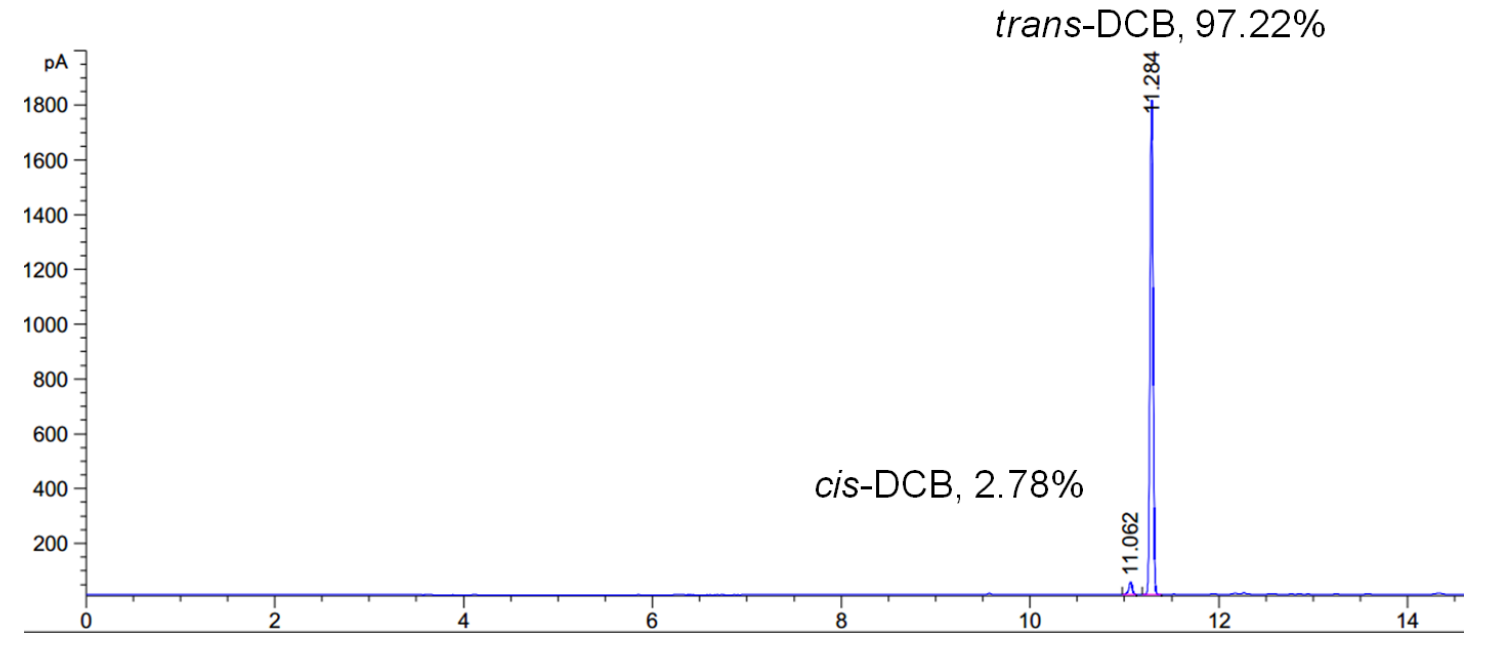

Figure S27. Relative uptake of cis-DCB and trans-DCB adsorbed in EtP6 $\beta$ after 24 hour using gas chromatography. 


\section{Recyclability of EtP6 $\beta$ Crystals}

An open $5 \mathrm{~mL}$ vial containing $0.10 \mathrm{~g}$ of 2(cis-DCB)@EtP6 was desolvated under vacuum at $150{ }^{\circ} \mathrm{C}$ overnight. The resultant crystals were charicterized by TGA and PXRD.

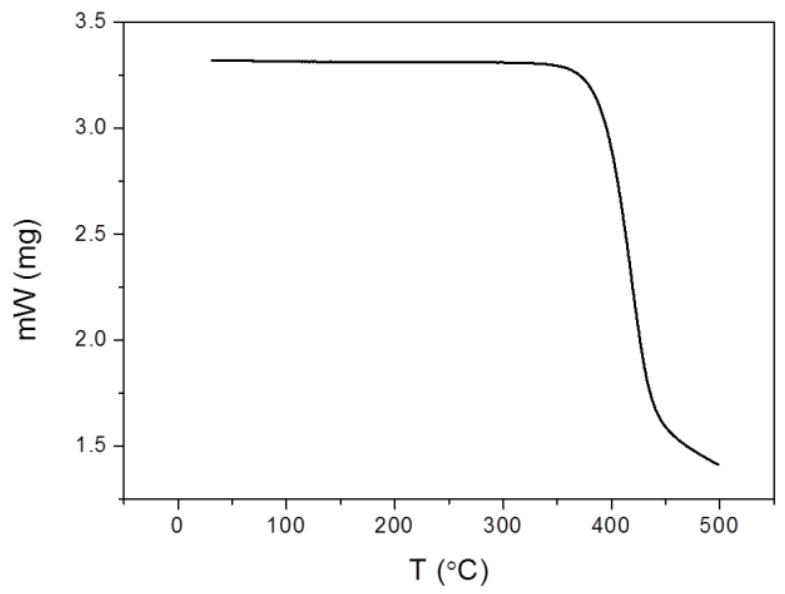

Figure S28. Thermogravimetric analysis of desolvated EtP6 upon removal of cis-DCB.

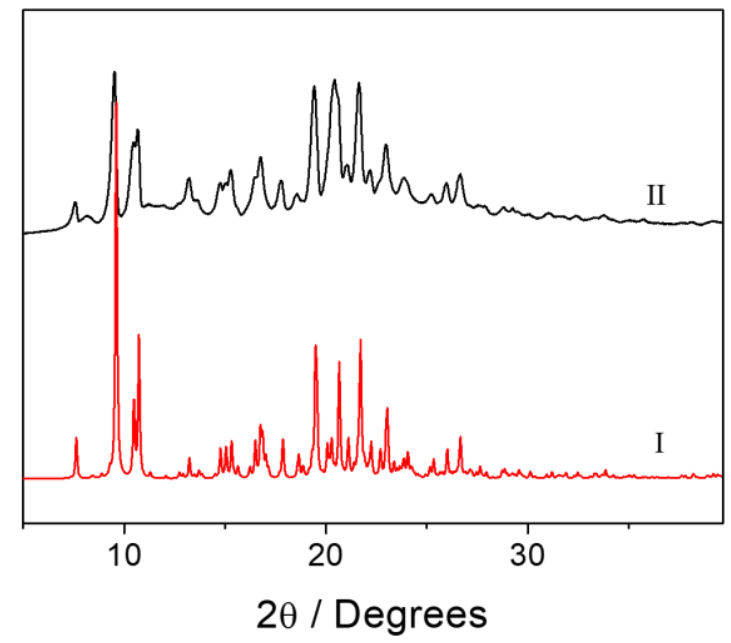

Figure S29. Powder X-ray diffraction patterns of EtP6: (I) EtP6 $\beta$; (II) desolvated trans-DCB@EtP6. This implies that upon removal of trans-DCB, trans-DCB@EtP6 will transform back to EtP6 $\beta$. 
trans-DCB, 96.7\%

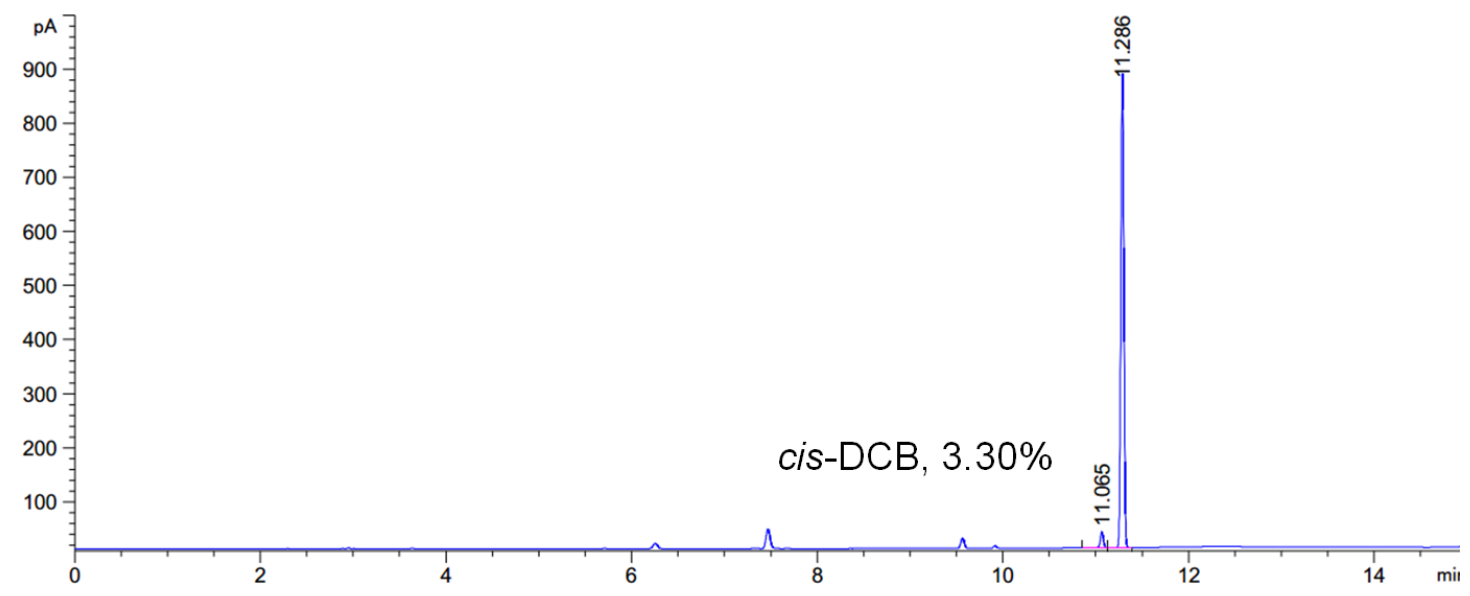

Figure 30. Relative uptake of $c i s-\mathrm{DCB}$ and trans-DCB adsorbed in EtP6 $\beta$ for 24 hours using gas chromatography after EtP6 was recycled 5 times. 


\section{References:}

S1 Hu, X.-B.; Chen, Z.; Chen, L.; Zhang, L.; Hou, J.-L.; Li, Z.-T. Pillar[ $n] \operatorname{arenes~(~} n=$ 8-10) with two Cavities: Synthesis, Structures and Complexing Properties. Chem. Commun. 2012, 48, 10999.

S2 Nowell, H.; Barnett, S. A.; Christensen, K. E.; Teat, S. J.; Allan, D. R. I19, the Small-Molecule Single-Crystal Diffraction Beamline at Diamond Light Source. $J$. Synchrotron Radiat. 2012, 19, 435.

S3 Sheldrick, G. M. (University of Göttingen, Germany) 2008.

S4 Sheldrick, G. M. Experimental Phasing with SHELXC/D/E: Combining Chain Tracing with Density Modification. Acta Cryst. Sect. D 2008, 66, 479.

S5 Sheldrick, G. M. SHELXT-Integrated Space-Group and Crystal-Structure Determination. Acta Cryst. Sect. A 2015, 71, 3.

S6 Sheldrick, G. M. A Short History of SHELX. Acta Cryst. Sect. A 2008, 64, 112.

S7 Sheldrick, G. M. Crystal Structure Refinement with SHELXL. Acta Cryst. Sect. C 2015, 71, 3 .

S8 Dolomanov, O. V.; Bourhis, L. J.; Gildea, R. J.; Howard, J. A. K.; Puschmann, H. OLEX2: a Complete Structure Solution, Refinement and Analysis Program. J. Appl. Cryst. 2009, 42, 339.

S9 Jie, K.; Liu, M.; Little, M. A.; Pulido, A.; Chong, S. Y.; Stephenson, A.; Hughes, A. R.; Sakakibara, F.; Ogoshi, T.; Blanc, F.; Day, G. M.; Huang, F.; Cooper, A. I. Near-Ideal Xylene Selectivity in Adaptive Molecular Pillar[n]arene Crystals. J. Am. Chem. Soc. 2018, 140, 6921. 